\title{
Advanced Materials and Convergence Technologies for Sustainable COVID-19 Healthcare Model
}

\author{
Anshuman Mishra ${ }^{1,2}$, Yogesh Shukla ${ }^{3}$, Ashutosh Tiwari ${ }^{1,2,3, *}$ \\ ${ }^{1}$ Institute of Advanced Materials, IAAM, Ulrika 59053, Sweden \\ ${ }^{2}$ VBRI, 7/16 Kalkaji Extension, New Delhi 110 019, India \\ ${ }^{3} \mathrm{mH}$ ospitals, 2/31 Nehru Enclave, New Delhi 110019, India \\ *Corresponding author: E-mail: director@iaam.se, Tel: (+46) 1313-2424
}

DOI: 10.5185/amlett.2021.011589

COVID-19 risk spread rapidly with several clinical manifestation and public health challenges. Research and innovation in advanced materials should be focused on solving critical problems associated with diagnosis, treatment and control models. Important challenges of COVID-19 public health system indicated to need of solutions based on cost, quality and universal availability in sustainable model. Standard healthcare models can be achieved by advanced technology of Artificial intelligence, loT, Database analytics and advanced materials more effectively for mass community. Next-generation ("smart") materials have capacity to contribute in theranostics models in better way with high accuracy in terms of performance and reliability. In summary, engineered nanoparticles and advanced technologies have translational potential. Being public health professionals, technologist and materials researchers involved in the prognosis research should develop certain priorities for the society.

In humans, COVID-19 risk spread rapidly with several clinical manifestation and public health challenges. On the basis of spread and severity, WHO characterized the COVID-19 situation as a pandemic [1,2]. Research and innovation in advanced materials in this prime time of COVID-19 pandemic should be focused on solving critical problems associated with diagnosis, treatment and control models. Most specifically stopping transmission and targeted drug therapy is major filed where materials researchers aims lie. Advanced technologies represent Nanomaterials more effectively in healthcare, for diagnosis and therapy [3]. The flexibility and compatibility of nano materials including quantum dots, graphene, silicon carbide (SiC) and carbon nanotubes etc.) and nanotechnology in public healthcare models, helps in its adoptability for medicine and industrial development [3-6]. The materials modified for the biosensor and bioelectronics field for better and unique performance in healthcare [7-9]. Technology make bridges between biological systems and electronic devices to monitor and control biological processes [8]. Therapeutic functional nanomaterials with combination of electronics demonstrates unconventional diagnostics capabilities [10]. Advanced technologies helpful to utilized materials in form of flexible battery, 3D integrated circuit, smart glass, biochips and G. fast chipset etc. for futuristic healthcare technologies [11].

The far-reaching implications of sustainable public healthcare models have so far been largely overlooked due to limitation in knowledge and resources at bottom level. Important challenges of COVID-19 public health system indicated to need of solutions for the diagnosis and treatment segment mostly for cost, quality and universal availability in sustainable model.

\section{Sustainable healthcare model}

Standard healthcare models can be achieved by advanced technology of Artificial intelligence, IoT, Database analytics and advanced materials more effectively for mass community [12]. The main agenda of standard and sustainable public health model is to understand and develop materials science and technology for different applications thus, realized that significantly different properties provide roadmap for nano-materials utilization and sensing mechanism for effective prognosis. Sustainability in advanced materials will always support any integration, which by conventional means connect materials to healthcare prognosis technologies by maintaining its characteristics at high efficiency. Integration of advanced technologies in materials field is most challenging in present time of COVID-19 pandemic, where difficulties in verifying their reliability, functionality and effectiveness judged. The efforts to build Sustainable healthcare model should take into account both what is available and what is required to control disease burden. Fig. 1 described about sustainable healthcare model for standard healthcare practices.

\section{Smart materials selection}

Next-generation ("smart") materials have capacity to contribute in targeted diagnosis and treatment in better way with high accuracy in terms of performance and reliability $[\mathbf{1 0 , 1 1}]$. While utilization of biomolecules (proteins, nucleic acids, and carbohydrates et.) for recognition and interaction with other bio components or biomolecules that are expressed in disease cells is helpful to make strategies 


\section{Advanced Materials Letters www.vbripress.com/aml}
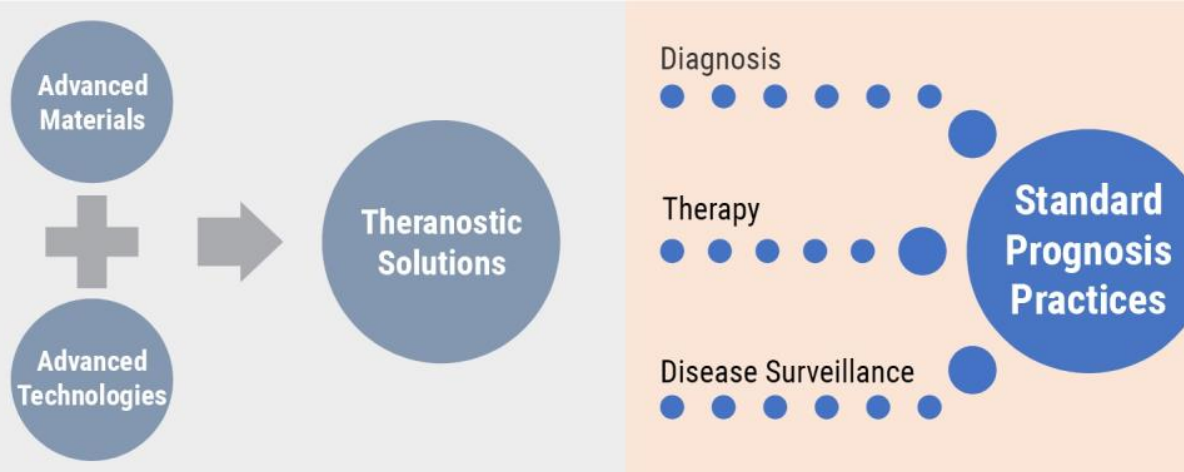

Fig. 1. Sustainable healthcare model for standard infectious diseases and healthcare practices.

for prognosis. Construction of quantum dots, nanoparticles, and 2D material platforms decorated with biological components such as antibodies, peptides, nucleic acid aptamers, carbohydrates, and folic acid are used extensively for prognosis model. The potential therapeutic candidates and materials bears potential mechanisms of action on COVID-19 [13,14]. Functional candidates and materials can be used for targeted therapy for COVID-19 upon validation and authorization. Further, materials engineering develop prototype and technologies based on streamline design and performance will be improved further with new materials to make better technologies with higher confidence in their properties.

\section{Improvement in technologies}

Advanced technologies are related to processing, optimization, manufacturing, refining methods will yield improved performance of healthcare products at lower cost.
The emergence of artificial intelligence (AI) and its progressively wider impact on many sectors requires an assessment of its effect on the achievement of the Sustainable Development Goals [12]. Cloud healthcare and AI have major impact of healthcare practices, while handling large community [15]. The primary skills with the design, implementation, and evaluation of an AI enabled healthcare systems revealed the importance of data science. In Fig. 2, processes for creating, implementing, and evaluating an AI enabled system for healthcare has been presented. Most importantly, opportunities of better technologies lie in fabrication, integration, and designing of materials for various healthcare developmental projects under one roof.

On the other hand, surveillance for monitoring and understanding disease progress in region and population is must for making control strategies. Disease surveillance uses information-based technologies for the collection,

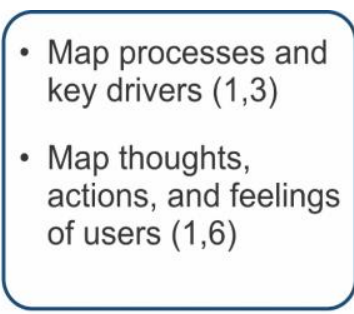

Understand the problem

- Interview and observe stakeholders $(1,3)$

- Analyze EHR data to understand clinical processes $(2,3,4)$
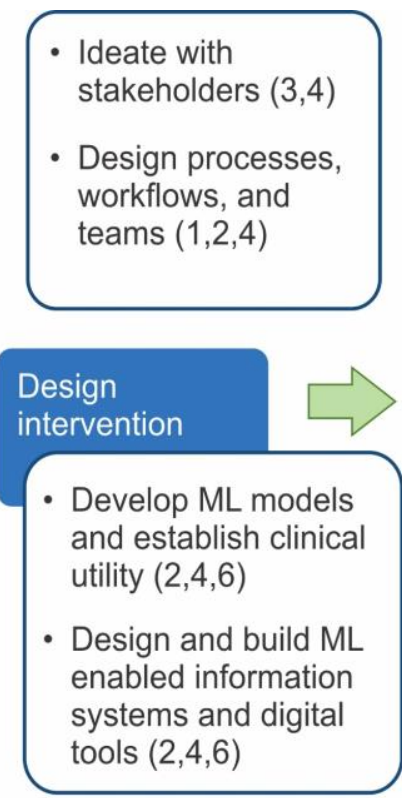

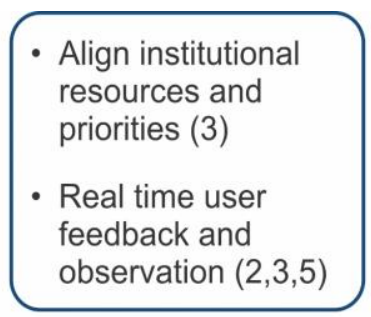

- Assess implementation, process, and clinical outcomes $(4,5,6)$

- Monitor ML model performance $(2,6)$

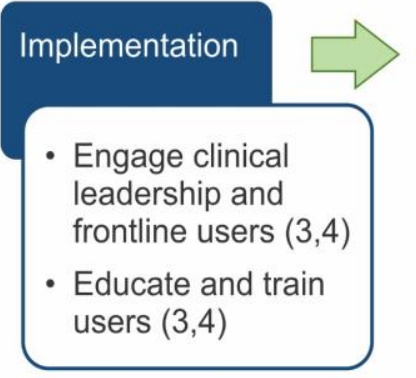

- Decide to iterate, maintain, scale, or retire intervention $(3,4)$

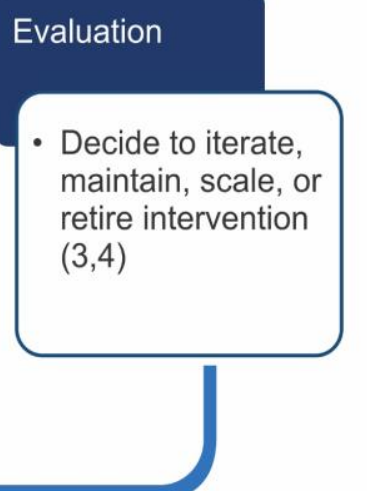

Fig. 2. Multidisciplinary process for creating, implementing, and evaluating an AI enabled system for healthcare (Adapted from [15]). 


\section{Advanced Materials Letters www.vbripress.com/aml}

analysis and interpretation of epidemiology data sets to evaluate the preventative health measures. Potential of disease surveillance helpful in early identification of the emergence of diseases outbreaks, which is further critical to the generation of timely responses [16].

Overall, improved and compatible technologies along with advanced materials research can better address public health challenges for mass healthcare.

\section{Theranostic solutions}

A theranostic agent is one which serves both diagnostic and therapeutic functions, a capability which will have obvious clinical advantages [17]. The theranostics, strategies are helpful in incorporating biomolecules with advanced materials via sensor-based technologies (image, light, sound or signal etc.) and used in diagnosis and therapy. Theranostic nanomedicines have promising intrinsic ability to confer imaging that may yield pharmacokinetic and biodistribution information for the presence of the exact molecular target before, during, and after therapy [18]. Multidisciplinary basic research with materials science, pharmacology, nanotechnology, medicine, biology, computational, IT, neuroscience and diversified expertise helps to frame personalized medicines for a broad range of diseases [17-19]. Finally, theranostic technologies have enormous potential to enhance current healthcare practices and contribute more in futuristic solutions.

\section{Practical implications}

Being public health professionals, technologist and materials researchers involved in the diagnosis and treatment research should develop certain priorities for the society. Since sustainable healthcare model for mass healthcare practices mainly drive by smart materials, advanced technologies $[\mathbf{1 6 , 1 9 - 2 4}]$. So, designed and development of expanding materials field keep pace with advancement in technologies with in information technologies, cloud healthcare, digital medicine and artificial intelligence for theranostic and disease surveillance services of COVID-19. Engagement in training and development of healthcare products against pathogenic information's for the safety of the society is logical shift in pandemic era.

\section{Conclusion}

In summary, engineered nanoparticles and advanced technologies have translational potential. The hope is that these strategies can persuade and advise those that are interested in the advanced materials field of healthcare to rationally design and develop prognosis models.

\section{Keywords}

Advanced materials, prognosis, artificial intelligence, theranostic technologies, COVID-19 pandemic.

Received:

Revised:

Accepted:

\section{References}

1. Hu, B.; Guo, H.; Zhou, P.; Shi, Z. L.; Nat. Rev. Microbiol., 2020 , https://doi.org/10.1038/s41579-020-00459-7.

2. Bedford. J. et al.; The Lancet, 2020, 395, 1015.

3. Tiwari, A.; Mishra, Y.K.; Kobayashi, H.; Turner, A.P.F. (Eds.) Intelligent Nanomaterials, 2nd Edition, 2016.

4. Satterfield, T.; Kandlikar, M.; Nature Nanotech., 2009, 4, 752.

5. Marschalek, I.; Hofer, M. et al.; Nature Nanotech. 2017, 12, 92.

6. Suvarnaphaet, P.; Pechprasarn, S.; Sensors, 2017, 17, 2161.

7. Yun, J.; Cho, H.Y. et al.; J. Mater. Chem. B, 2020, 8, 7303.

8. Bolotsky, A.; Butler, D.; et al., ACS Nano, 2019, 9, 9781.

9. Kim, J.; Campbell, A.S.; et al., Nat. Biotechnol. 2019, 37, 389.

10. Tiwari, A.; Uzun, L.; (Eds.), Advanced Functional Materials, 2015.

11. Tiwari, A.; (Ed.), Advanced Healthcare Materials, 2014.

12. Ting, D.S.W.; Carin, L.; Dzau, V.; Wong, T.Y.; Nat. Med., 2020, 26, 459.

13. Mishra, A.; Patra, S.; Shukla, S. et al.; Adv. Mater. Lett., 2020, 11, 20041494.

14. Tang, Z.; Kong, N.; et al., Nat. Rev. Mater., 2020, 5, 847.

15. Li, R. C.; Asch, S. M.; Shah, N. H.; npj Digit. Med., 2020, 3, 107.

16. Vinuesa, R., Azizpour, H., Leite, I. et al. Nat Commun., 2020, 11 , 233.

17. Tiwari, A.; Patra, H.K.; Choi, J.W.; Advanced Theranostic Materials, 2015.

18. Ashokan, A.; Somasundaram, V.H.; Gowd, G.S. et al.; Sci. Rep. 2017, 7, 14481.

19. Patra, H.K.; Khaliq, N. U.; Romu, T. et al.; Adv. Healthcare Mater., 2014, 3, 526.

20. Parlak, O.; Incel, A. et al.; Biosens. Bioelectron., 2017, 89, 545.

21. Patra, H.K.; Sharma, Y. et al.; Nanoscale, 2016, 8, 17213.

22. Mei, X.; Lee, H.C. et al.; Nat. Med., 2020, 26, 1224.

23. Tiwari, A.; Tiwari A.; Nanomaterials in Drug Delivery, Imaging, and Tissue Engineering, 2013.

24. Satija, S.; Mehta, M. et al.; Future Med. Chem. 2020, 12, 1607. 